\title{
A fern WUSCHEL-RELATED HOMEOBOX gene functions in both gametophyte and sporophyte generations
}

\author{
Christopher E. Youngstrom, Lander F. Geadelmann, Erin E. Irish and Chi-Lien Cheng ${ }^{*}$
}

\begin{abstract}
Background: Post-embryonic growth of land plants originates from meristems. Genetic networks in meristems maintain the stem cells and direct acquisition of cell fates. WUSCHEL-RELATED HOMEOBOX (WOX) transcription factors involved in meristem networks have only been functionally characterized in two evolutionarily distant taxa, mosses and seed plants. This report characterizes a WOX gene in a fern, which is located phylogenetically between the two taxa.

Results: CrWOXB transcripts were detected in proliferating tissues, including gametophyte and sporophyte meristems of Ceratopteris richardii. In addition, CrWOXB is expressed in archegonia but not the antheridia of gametophytes. Suppression of CrWOXB expression in wild-type RN3 plants by RNAi produced abnormal morphologies of gametophytes and sporophytes. The gametophytes of RNAi lines produced fewer cells, and fewer female gametes compared to wild-type. In the sporophyte generation, RNAi lines produced fewer leaves, pinnae, roots and lateral roots compared to wild-type sporophytes.
\end{abstract}

Conclusions: Our results suggest that CrWOXB functions to promote cell divisions and organ development in the gametophyte and sporophyte generations, respectively. CrWOXB is the first intermediate-clade WOX gene shown to function in both generations in land plants.

Keywords: Apical cells, Ceratopteris richardii, Fern, Gametophyte and sporophyte, Meristem, RNAi, WUSCHELRELATED HOMEOBOX (WOX), Stem cell

\section{Background}

Stem cells are self-renewing pluripotent cells. In vascular plants, they are located in the shoot apical meristem (SAM) and root apical meristem (RAM). Stem cells divide at a low frequency to produce daughter cells that will either maintain the stem cell pool or actively divide and take on new identities to form new organs $[1,2]$. The size of a stem cell population varies among different species and is strictly maintained as a part of the meristem $[3,4]$. Failure to coordinate multiple inter- and intra-cellular signals disrupts development and results in altered plant body architecture $[4,5]$. In addition to hormonal signals, inter-cellular signaling is mediated by small peptide ligands and their cognate receptors. These

\footnotetext{
* Correspondence: chi-lien-cheng@uiowa.edu

Department of Biology, University of lowa, 129 E. Jefferson St., lowa City, lowa 52242, USA
}

signals converge to regulate specific transcription factors to achieve a balance among the populations of stem cells, the faster dividing cells, and the differentiating cells of the meristem [6, 7]. In Arabidopsis thaliana, the homeobox transcription factor WUSCHEL (WUS) is a key player in shoot meristem maintenance; WUS expression is transcriptionally regulated and the protein acts non-cell-autonomously by moving from the organizing center $(\mathrm{OC})$ to the central zone $(\mathrm{CZ})$ of the SAM to both activate and repress gene transcription in order to maintain meristem cells in a pluripotent state [8].

WUS belongs to the family of WUSCHEL-RELATED $H O M E O B O X$ (WOX) transcription factors, which are characterized by the presence of a conserved homeodomain $[9,10]$. Phylogenetic analyses of land plant WOX genes group members into three clades: ancient, intermediate, and modern ( [11] Additional file 1: Figure S1). The progenitor of $W O X$ genes existed in the last

(c) The Author(s). 2019 Open Access This article is distributed under the terms of the Creative Commons Attribution 4.0 International License (http://creativecommons.org/licenses/by/4.0/), which permits unrestricted use, distribution, and 
common ancestor of land plants and green algae and, through successive gene duplication and functional diversification, gave rise to the three clades of $W O X$ genes $[9,12]$. All land plants that have been examined, nonvascular and vascular alike, possess WOX genes of the ancient clade, while the intermediate clade only exists in vascular plants, and the modern clade is found in seed plants and ferns, but has not been found in lycophytes $[13,14]$. Based on the presence of two subgroups of the intermediate clade in the lycophytes and sequence relatedness of only one subgroup to the modern clade WOX genes, it has been proposed that the intermediate subgroup shared a progenitor with the modern clade $[11,14]$. The modern clade, or the WUS clade, has experienced further expansion in seed plants as Picea abies possesses five and $A$. thaliana possesses eight WUS clade members $[9,15]$, compared to the single member found in the fern, Ceratopteris richardii [11]. Modern clade WUS proteins contain, in addition to the canonical homeobox, the WUS box (TL-LFPMILV) [9]. Both conserved domains are required for meristem maintenance in the A. thaliana SAM [16]. Maintenance of the SAM [17] and RAM $[18,19]$ is under the control of AtWUS and AtWOX5, respectively, and in addition, AtWOX4 functions in the vascular cambium stem cells $[20,21]$ where continually dividing cells produce phloem and xylem during secondary growth. All other AtWOX genes of the three clades play roles in early embryo development or in organ development, including leaf, root, and floral organs [12, 21-28].

The WOX gene family has been widely studied in land plants, including $C$. richardii, but functional studies are limited to seed plants such as A. thaliana (e.g. $[9,16]$, ), Oryza sativa (e.g. [29],), P. abies [30, 31] and moss Physcomitrella patens [32]. Five WOX genes, CrWOX13A and $C r W O X 13 B$ of the ancient clade, CrWOXA and $C r W O X B$ of the intermediate, and CrWUL of the modern clades have been identified in $C$. richardii [11]. RT-PCR results showed that $\mathrm{Cr} W O X 13 \mathrm{~A}$ and $\mathrm{Cr} W O X B$ are equally expressed in all tissue examined, including root tip, gametophyte, and young sporophyte; whereas CrWOXA was expressed more strongly in the root tip and CrWUL in the root tip and gametophyte [11]. In situ hybridization analyses of the latter two genes showed localized expression. CrWOXA is expressed in the root apical cell and in the lateral root apical cell. In addition to expression in the vascular bundle of the leaves [33], CrWUL mRNA is localized to the cutting edge of the lateral root apical cell which divides asymmetrically, proximal to the main root axis [11]. CrWOXB shows a broad expression pattern in the root tip, consistent with the high levels of expression detected by RT-PCR [11].

The unbiased expression of $\mathrm{CrWOX} 13 \mathrm{~A}$ and $\mathrm{Cr} W O X \mathrm{~B}$ in both gametophyte and sporophyte generations presents an opportunity to understand the ancestral functions of WOX proteins. In the moss $P$. patens, only the ancient clade of $W O X$ genes exists, and, in contrast to A. thaliana ancient WOX genes, $P$. patens ancient $W O X$ genes function in both generations [32]. Only two AtWOX genes, AtWOX2 and AtWOX8, are expressed in both sporophyte and gametophyte generations [25]; all other AtWOX genes seem to function only in the sporophytes [12, 21-28]. Interestingly, in Nicotiana tabacum, transcripts of two ancient and one intermediate WOX genes are found in both the gametophyte and sporophyte tissues [34].

Sister clade to seed plants, ferns have sporophytic SAMs that are composed of multiple zones resembling that of the seed plants $[35,36]$. How these zones are involved in stem cell maintenance and organ initiation is unclear. Moreover, how the fern gametophyte notch meristem is maintained is completely unknown. Thus, WOX genes provide an entry point for understanding the meristem of ferns at both the development and the evolution levels. So far, the combination of WOX gene family evolution and their developmental functions has only been studied in detail in the moss P. patens and angiosperms. Similar investigation in the fern will bridge the gap in our knowledge of meristem evolution. Furthermore, comparison between the gametophyte and the sporophyte meristems within the fern will provide insight into the co-option of gene network between the meristem of the two generations. This understanding can only be fully realized with the expression and functional analyses of all five WOX fern genes. Here, we present the completed study of one of the five WOX genes found in C. richardii, $C r W O X B$, which is expressed in both sporophyte and gametophyte generations [11], to examine its expression in the meristems of sporophyte shoot and gametophyte using sectioned and whole mount in situ hybridization, respectively. The possible function of $\mathrm{CrWOXB}$ in both generations was examined by RNAi suppression of $\mathrm{Cr} W O X B$ expression in transgenic $C$. richardii plants. These results show that $C r W O X B$, an intermediate-clade WOX gene, is expressed in regions of cell proliferation in both the gametophyte and sporophyte. The phenotypes of the RNAi suppression lines were consistent with meristem defects, providing the first demonstration of WOX gene function in a fern.

\section{Results}

CrWOXB is expressed in regions of cell division in both the gametophyte and sporophyte generations

The expression of $\mathrm{Cr} W O X B$ was observed in developing gametophytes and in sporophyte leaves. $\mathrm{Cr} W O X B$ was expressed at low but discernable levels before ( $\mathrm{d} 8$ and d10) sexual maturation and increased in gametophytes 


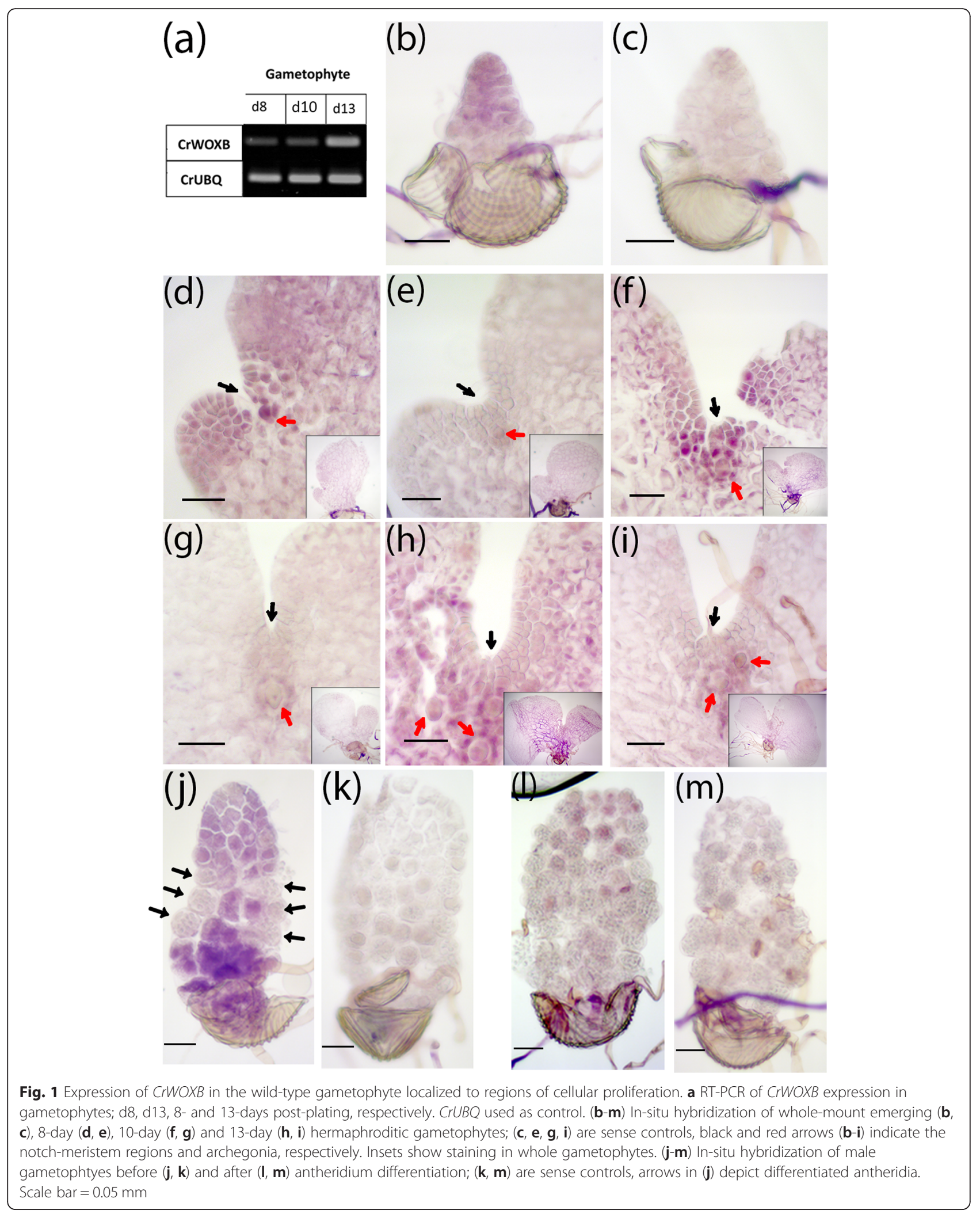




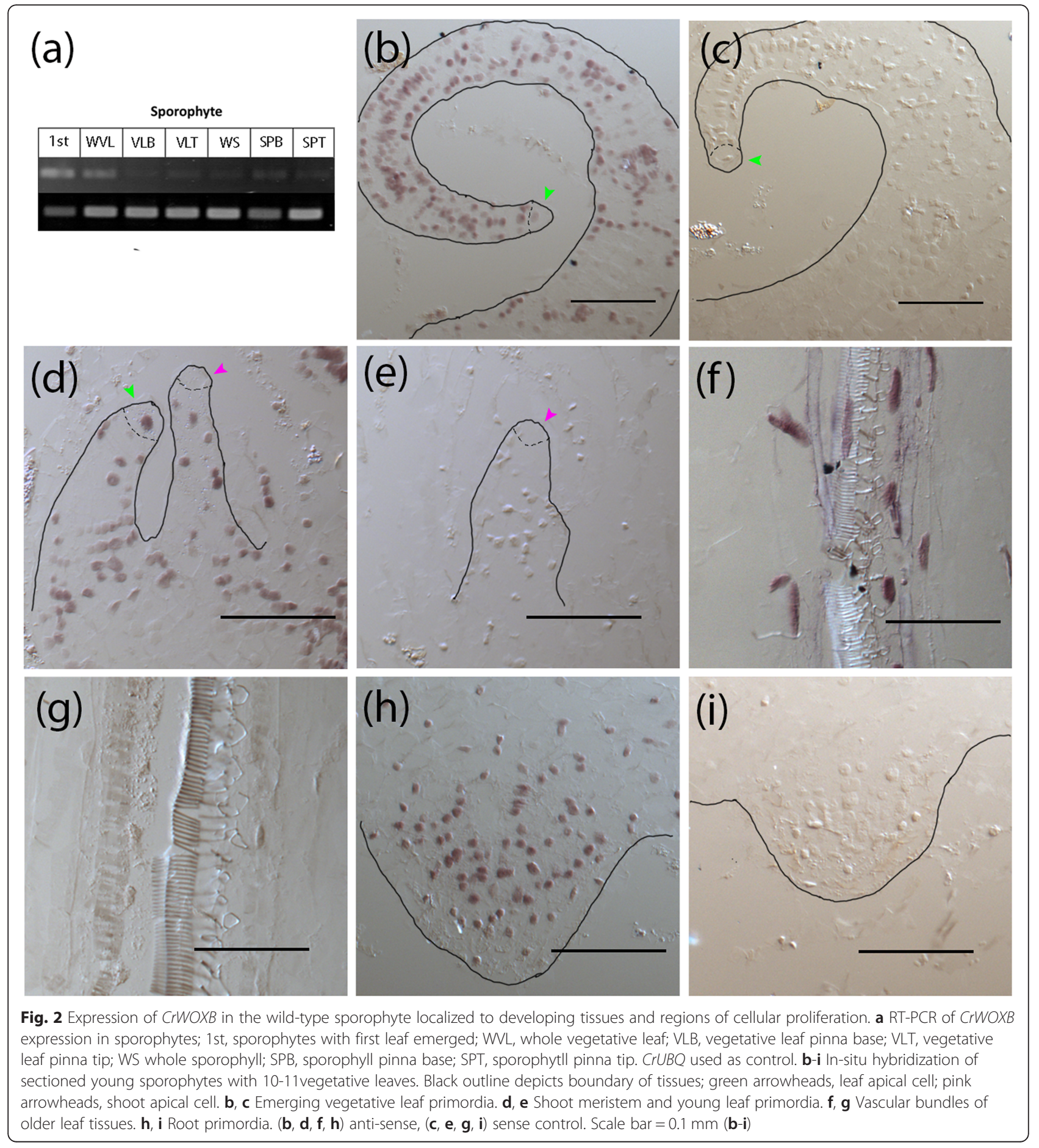

at sexual maturity (d13) (Fig. 1a). Whole-mount in situ hybridization revealed that $C r W O X B$ mRNA was expressed in recently germinated gametophytes (Fig. 1b, c) and then in the notch meristem region in gametophytes before (d8, Fig. 1d, e; day 10, Fig. 1f, g) and after (d13, Fig. 1h, i) sexual maturation. Consistent with increased expression at $\mathrm{d} 13$ detected by RT-PCR analysis (Fig. 1a), d13 gametophytes showed a broader area of
CrWOXB expression than $\mathrm{d} 8$ and $\mathrm{d} 10$, that was also further away from the notch region. In addition, $\mathrm{Cr} W O X B$ expression was also detected in developing archegonia (Fig. 1d, f red arrows) suggesting a role in organ specification in hermaphrodites. At d8, similar to hermaphrodites, male gametophytes expressed $\mathrm{CrWOXB}$ in cells prior to antheridia development (Fig. 1j). The expression declined in $\mathrm{d} 13$, when most cells have developed into antheridia 


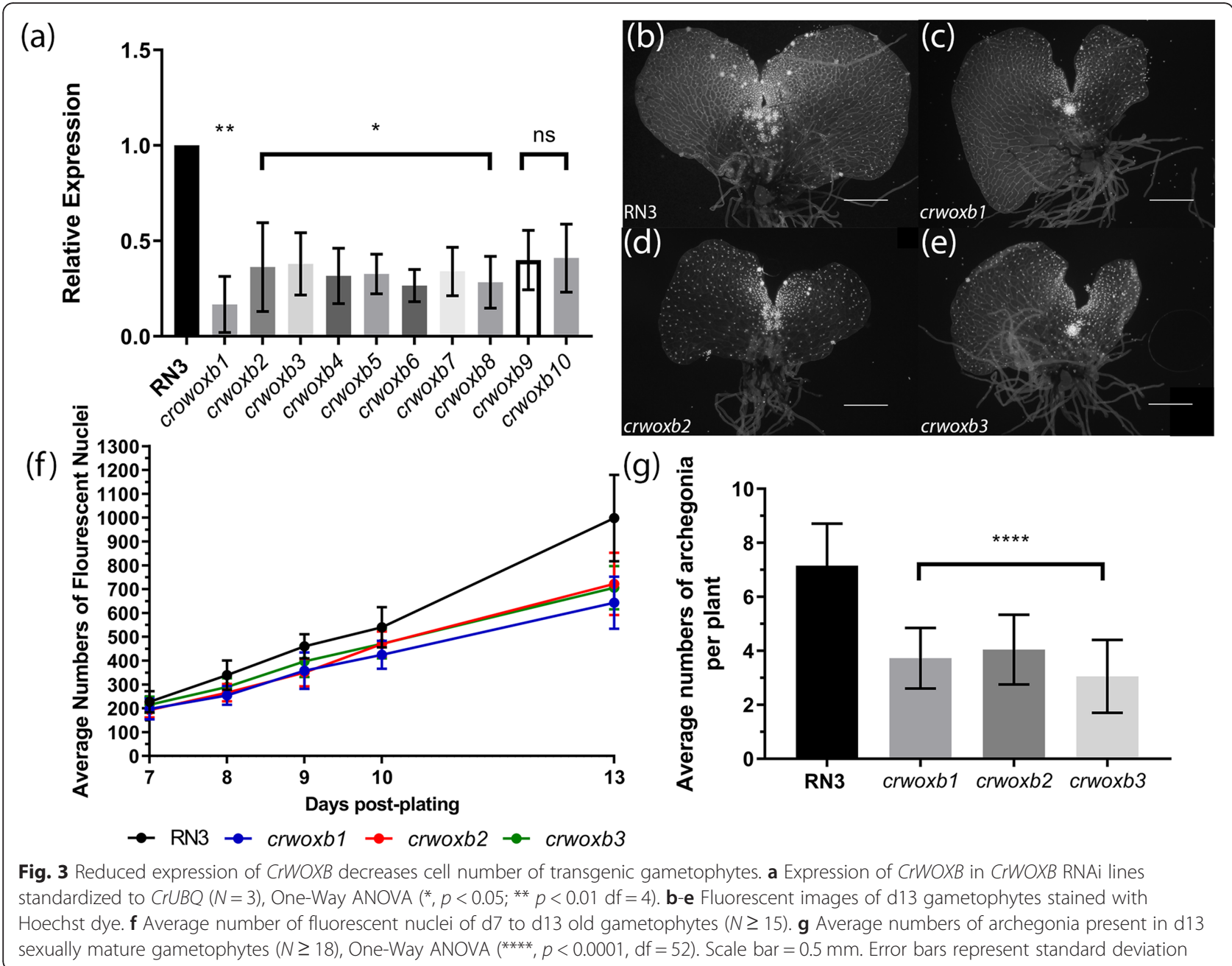

(Fig. 11). Thus, the expression of $\mathrm{Cr} W O X B$ in gametophytes is in actively dividing region in both hermapthrodites and males and the notch meristem and the archegonium of hermaphrodites.

The first set of leaves (total of 16-21 leaves) formed by $C$. richardii sporophytes are vegetative (do not produce sporangia), followed by an indeterminate number of sporophylls (produce sporangia). Pinnae refer to the leaflets of both vegetative and sporophyll leaves. The expression of $\mathrm{Cr} W O X B$ in sporophyte tissues detected by RT-PCR showed the highest expression in sporophytes with one fully expanded leaf and in the later vegetative leaves but was barely detectable in the sporophylls, the pinnabase and tip of the vegetative leaves (Fig. 2a). CrWOXB mRNA was detected by in situ in young vegetative leaves (Fig. 2b), the shoot tip (Fig. 2d), leaf vascular bundles (Fig. 2f), and in root primordia (Fig. 2h) of young sporophytes bearing 10-11 vegetative leaves, when the first visible fiddlehead was observed. Notably $\mathrm{Cr} W O X B$ expression is seen within the apical cells of leaf primordia and more mature leaf (Fig. 2b, d, green arrowhead) but not in the apical cell of the SAM (Fig. 2d, purple arrowhead). Thus, $C r W O X B$ expression is localized to regions of actively dividing cells in the sporophyte generation.

\section{CrWOXB is required for proper growth of gametophytes}

To carry out functional analyses, we created $C r W O X B$ RNAi suppression, crwoxb, lines, by using Agrobacterium-mediated transformation of gametophytes. Suppression of $C r W O X B$ transcripts were quantified in young sporophytes with 6-7 fully expanded vegetative leaves by RT-qPCR. CrWOXB expression in $\mathrm{T}_{2}$ sporophyte of crwoxb lines were found to express a range of levels from a high average of $\sim 40 \%$ in crwoxb10 to $~ 16 \%$ in crwoxb1 compared to wild-type plants (Fig. 3a). The variation is likely due to position effect of the transgene [37]. No plants were recovered with undetectable CrWOXB expression.

Consistent with expression in the meristem and other regions of cell division (Fig. 1b-h), d13 
gametophytes of crwoxb lines were smaller and had an altered morphology, including a wider notch (Fig. 3b-e; Additional file 2: Figure S2a-d inset) between the two lobes of the thallus. The wider notch appeared to be the result of a localized combination of fewer trapezoidal meristem cells and altered cell division planes, which prevented the lobes of the gametophyte from growing together. To quantify the gametophyte size, gametophyte nuclei were stained and counted. Wild-type and crwoxb lines produced similar numbers of gametophyte cells prior to $\mathrm{d} 8$. After $\mathrm{d} 8$, development of crwoxb lines is delayed by 1 day (Fig. 3f; Additional file 5: Table S2). The gametophyte notch meristem is generally formed from $\mathrm{d} 7$ to $\mathrm{d} 8$. The average numbers of cells produced by crwoxb gametophytes were less than wild-type gametophytes and the difference increased with time (Fig. 3f; Additional file 5: Table S2).

Archegonia house the eggs and are direct derivatives of the notch meristem in C. richardii [38]. Because the gametophytes of crwoxb lines had fewer cells, we hypothesized that they would also develop fewer archegonia. To test this, we compared numbers of archegonia in wild-type and the crwoxb lines and found that indeed the $c r w o x b$ lines produced fewer archegonia than wildtype plants (Fig. 3g; Additional file 6: Table S3). The archegonia of the crwoxb lines were functional as they produced sporophytes. The reduction in archegonia numbers could be due to fewer cells of crwoxb gametophytes or to involvement of CrWOXB in the specification of archegonia progenitor cells. To distinguish between these two possibilities, we compared the number of archegonia to number of cells in the entire gametophyte of wild-type and crwoxb lines (Additional file 6: Table S3). Thirteen-day-old crwoxb gametophytes, although having fewer cells, had on average 55 more, not fewer, cells for each archegonium than wild-type gametophytes. This result rules out the first but not the second scenario.

\section{CrWOXB promotes leaf development in the sporophyte generation}

In situ hybridization revealed localized expression of $C r W O X B$ in leaf primordia and developing leaves in the sporophyte (Fig. 2b, d), consistent with a role of leaf initiation. These results prompted an examination of leaf initiation and development in the crwoxb lines. In the wild type, 16-21 vegetative leaves are formed, followed by sporophylls (Fig. 4e). In contrast, crwoxb lines initially produced fewer vegetative leaves (Fig. 4e) before producing sporophylls. The number of sporophylls produced seemed to be comparable to the wild-type as both continue producing sporophylls indefinitely; however, the number of pinnae of each sporophyll was greatly reduced compared to that of wild-type plants (Fig. 4f).
These results indicate a role of CrWOXB in leaf morphogenesis during both the vegetative and reproductive phases of the sporophyte generation.

\section{CrWOXB promotes root and lateral root initiation during sporophyte development}

The expression of $C r W O X B$ in root primordia (Fig. 2h) and during lateral root formation suggested a role for CrWOXB in root initiation and/or development. Sporophytes with 6-7 fully expanded leaves were grown in liquid media for 14 days before root observation. Wildtype sporophytes (Fig. 5a) exhibited more roots and were more branched than crwoxb lines (Fig. 5b-d). When quantified, the average number of roots (Fig. 5e) and lateral roots per plant (Fig. 5f) were significantly reduced in crwoxb lines compared with wild-type plants. Similar to leaf initiation and development, the significant reduction of root and lateral root numbers further confirmed the role of CrWOXB in organ initiation.

\section{Discussion}

The WOX genes, especially the modern clade member WUS, are well studied in angiosperms. Considering their important roles in meristem maintenance, how these genes function in the ferns will help the understanding of fern meristems and their maintenance. Here we presented the first functional analysis of a fern $W O X$ gene, $C r W O X B$, and showed its role in both gametophyte and sporophyte generations.

\section{CrWOXB functions in both the gametophyte and sporophyte generations}

Reduced cell divisions in hermaphroditic gametophytes of crwoxb lines suggested that CrWOXB promotes cell division, mirroring intermediate WOX proteins in $A$. thaliana and $P$. abies where these proteins activate cyclin genes, which regulate the progression of the cell cycle $[30,39,40]$. In addition to CrWOXB's function in cell division, in the hermaphrodites CrWOXB also seemed to play a role in specifying cells to become archegonia (Fig. 1g; Additional file 6: Table S3) in the region where $C r W O X B$ is expressed highly. The reduced number of archegonia in crwoxb lines could be explained by noncell autonomous action of CrWOXB, where decreased expression in crwoxb lines would need more cells to produce some threshold concentration for specification. Once specified, the egg-cell develop normally: its maturation and embryo development were unaffected in crwoxb lines, based on the observation that sporophytes formed after 5 days post-fertilization in both wild-type and crwoxb gametophytes. In the male, cell proliferation is followed closely by differentiation into antheridia [41, 42]. We detected $C r W O X B$ in cells before, but not after 

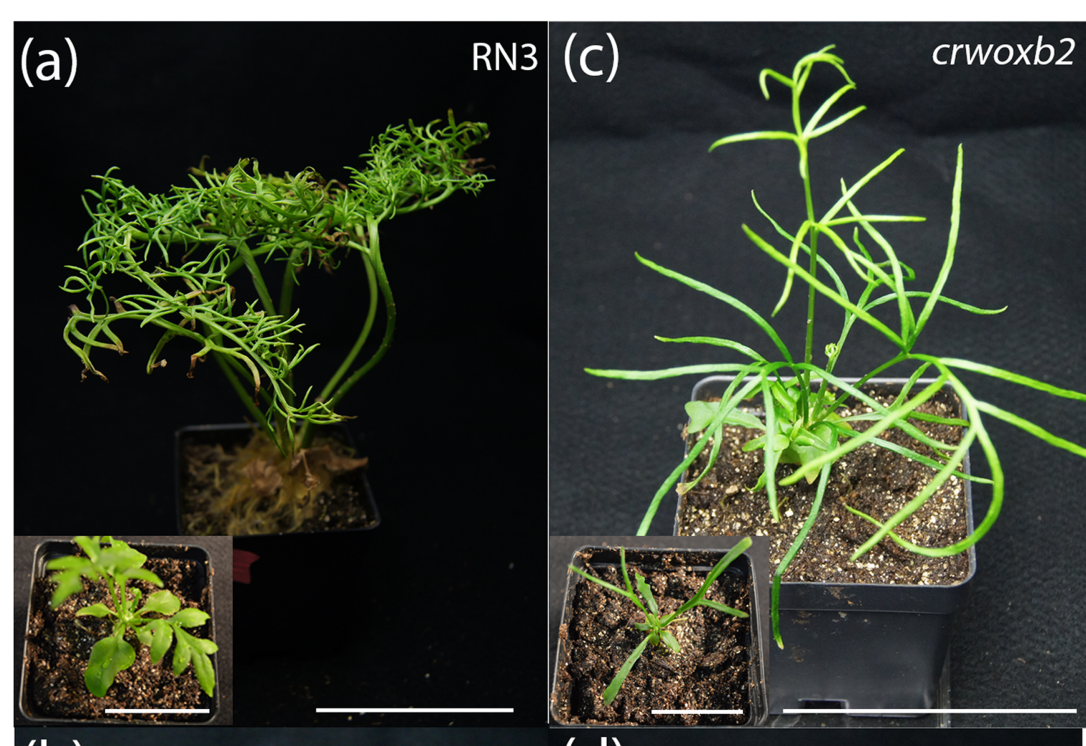

(b)
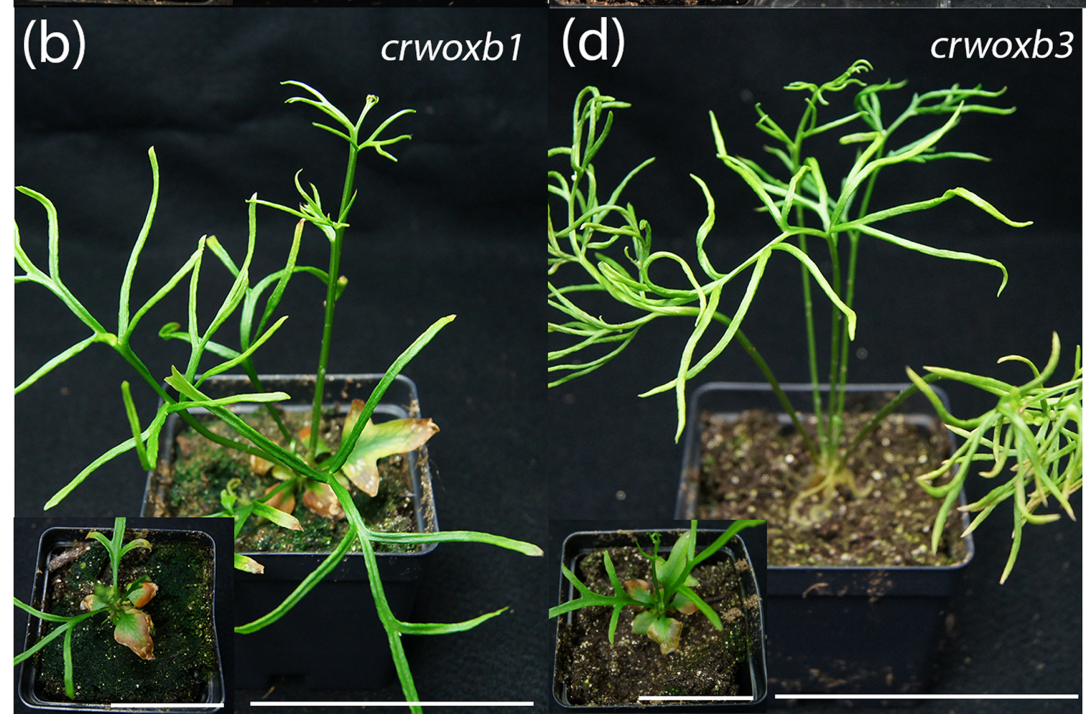

(e)

(f)
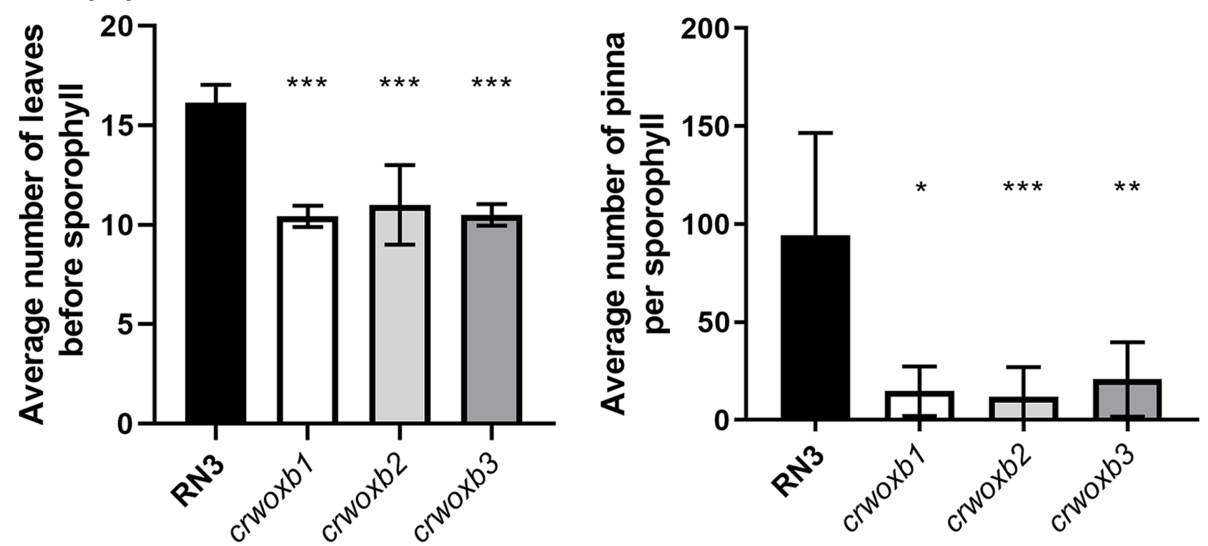

Fig. 4 Transgenic sporophytes produce simpler leaves. a-f Sexually mature sporophytes producing sporophylls. RN3 are wild-type plants. (a-d inset) Young sporophytes of lines depicted in a-d. e Average numbers of leaves produced before the first sporophyll ( $N \geq 6$ plants), One-Way ANOVA (***, $p<0.001)$. f Average numbers of pinna per sporophyll $\left(N \geq 11\right.$ fronds). Scale bar $=9.3 \mathrm{~cm}$. One-Way ANOVA $\left({ }^{*}, p<0.05 ;{ }^{* *}, p<0.01\right.$; $\left.{ }^{* * *}, \mathrm{p}<0.001 \mathrm{df}=24\right)(\mathbf{a}-\mathbf{d})$. Scale bar $=4.65 \mathrm{~cm}(\mathbf{a}-\mathbf{d}$ inset). Error bars represent standard deviation 

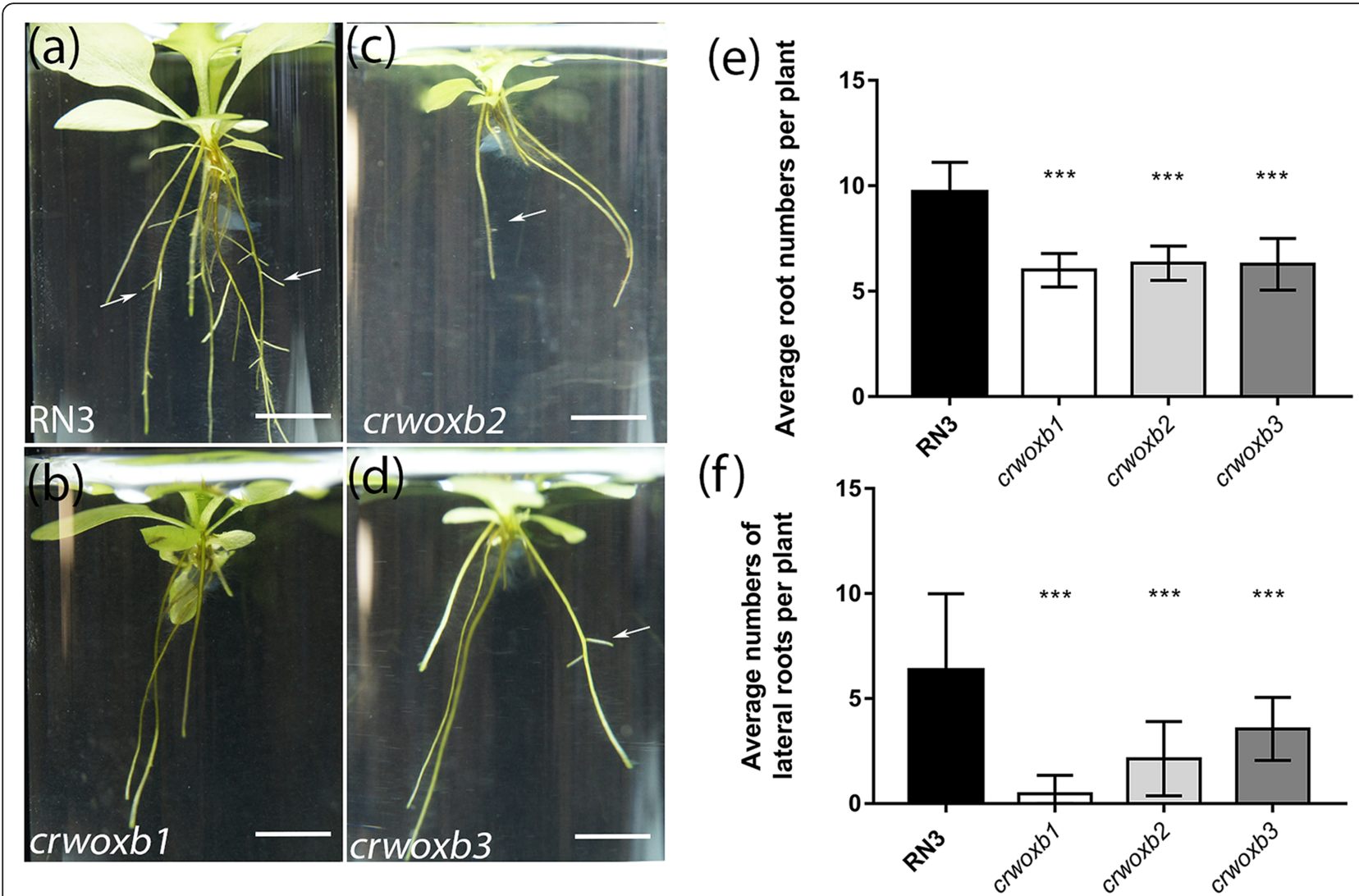

Fig. 5 Transgenic sporophytes produce fewer roots and lateral roots. (a-d Images of lateral root growth from wild-type and transgenic plants grown for 2 weeks in liquid culture (white arrows show lateral roots). e Average number of roots per plant ( $N \geq 14$ sporophytes from each line). $\mathbf{f}$ Average numbers of lateral roots per plant ( $\mathrm{N} \geq 14$ sporophytes from each line). e, f One-way ANOVA $(* * *, p<0.0001 \mathrm{df}=86)$. Scale bar $=8 \mathrm{~mm}$. Error bars represent standard deviation

differentiation into antheridia in $\mathrm{d} 8$ gametophytes (Fig. 1j, l).

In the sporophyte generation, abnormal phenotypes were observed in both the shoot and the root of crwoxb lines. In the shoot, the number of sterile fronds and pinna of the fertile fronds was diminished and similarly in the root, both root and lateral-root numbers were decreased. Therefore, we conclude that CrWOXB functions to promote cell division and possibly to specify organ formation in both generations of $C$. richardii.

$C r W O X B$ functions in both generations, whereas its ortholog in A. thaliana, AtWOX9, has only been shown to function in sporophytes [27, 43]. Prior to this work, only the ancient clade of WOX genes have been shown to function in both the gametophyte and the sporophyte generations of $P$. patens [32]. The trend of diminishing WOX gene function in the gametophytes during evolution is consistent with the comparative transcriptome profiling between the moss Furnaria hygrometrica and A. thaliana, in which an enrichment of bryophyte gametophyte-biased transcription factors are found in sporophyte-biased (and sporophyte-specific) A. thaliana orthologs [44].

\section{The role of CrWOXB in the meristems of gametophytes and sporophyte}

In the gametophyte, $C r W O X B$ was expressed in both the male and hermaphrodite. Expression in the male persisted briefly, during thallus growth, before cells differentiate into antheridia. Similarly, in the hermaphrodite, $C r W O X B$ was expressed shortly after spore germination; however, its expression appeared in the notch region, during and after the emergence of the lateral meristem. The expression pattern of $C r W O X B$ in the male and hermaphrodite is in agreement with cell proliferation regions delineated by [42]. Our results suggest that $C r W O X B$ function is required soon, if not immediately, after spore germination.

All growth in $C$. richardii sporophytes, as in other ferns, can be traced back to single, apical cells [45]. The shoot apical cell of $C$. richardii sits atop a slender stalk of meristem cells in a region defined as the proliferation 
zone [36]. Leaf initiation commences with the specification of one of the peripheral shoot meristem cells as a leaf apical cell, which persists throughout leaf development $[46,47]$. Roots similarly are formed by the persistent action of a root apical cell [48]. Expression of $C r W O X B$ is homogenous in the shoot, root, leaf primordia, and the vascular tissues signifying a more general role of CrWOXB in each region of cell proliferation. The homogenous expression pattern of $C r W O X B$ in the primordia is similar to what was observed of $A t W O X 9$ in Arabidopsis shoot apical meristem $[9,40]$. The expression pattern of $C r W O X B$ in the root primordia is similar to that in the mature root tip reported by Nardmann et al. [11], and is expressed in tissues outside of where CrWOXA, the other intermediate CrWOX gene, is expressed, suggesting some functional divergence between these two paralogs. Interestingly, $\mathrm{Cr} W O X B$ expression was not detected in apical cell of the shoot and root but was observed in the leaf primordia (Fig. 1h). We have consistently observed this difference, but the significance is unclear. The $C r W O X B$ may be regulated differently in the shoot and root apices and in the leaf primordia.

It may not be surprising to find that $C r W O X B$ played a role in both gametophytes and sporophylls. As observed by Hagemann [60], the pinnae of sporophyll and the gametophytes of the ferns are of structural similarity as both are dorsiventral with marginal meristematic growth and produce abaxial reproductive organs.

\section{The relationship between CrWOXB and the intermediate WOX transcription factor family}

In A. thaliana, the homeodomain of AtWOX8 and AtWOX9 can partially rescue meristem function in a wus-1 background, which establishes the intermediate WOX homeodomain as a key motif for meristem function [16]. Outside of the homeodomain, seed plant intermediate WOX members contain conserved N-terminal and $\mathrm{C}$-terminal motifs that are not shared with intermediate clade proteins in $C$. richardii, CrWOXA and CrWOXB (Additional file 3: Figure S3). Despite the divergence outside the homeodomain, phenotypes of crwoxb in the shoot and root are reminiscent of AtWOX9 null-mutant seedlings which fail to form leaves, secondary shoots and lateral roots [27]. The presence of the $\mathrm{C}$-terminal domain and $\mathrm{N}$-terminal motifs may be required for embryo patterning and development of the suspensor in A. thaliana because, in a complementary experiment, AtWUS, which does not contain the $\mathrm{N}$ - and C-terminal motifs of the intermediate clade, cannot rescue embryo arrest in Atwox8 Atwox9 double mutants [16]. Therefore, in C. richardii, the homeodomain is the most likely motif involved in cell proliferation and organ specification in the gametophyte and sporophyte, while the divergent $\mathrm{N}$ - and $\mathrm{C}$ - terminal sequences may contain yet-to-be recognized motifs that have additional function during embryogenesis.

\section{Conclusion}

We have functionally characterized an intermediate clade WOX protein CrWOXB throughout gametophyte and sporophyte development in the fern model $C$. richardii and found that $C r W O X B$ is expressed in proliferating tissues of both generations. Knockdown crwoxb lines produce fewer gametophyte cells, and smaller sporophytes with fewer sporophyte organs, suggesting a conserved function in gametophytes and sporophytes despite their different architecture. Methods and results presented here serve as model for the analysis of the remaining $W O X$ genes in $C$. richardii in order to understand how this gene family has diversified its functions in proliferative regions of the gametophyte and sporophyte generations.

\section{Methods}

\section{Plant growth conditions}

Spores of C. richardii strain Rn3 (wild-type) were originally obtained from Carolina Biological Supply Company (Burlington, NC). Wild-type and CrWOXB RNAi suppression lines (crwoxb) were surface sterilized in $4 \%$ sodium hypochlorite and $0.5 \%$ Tween-20 for $5 \mathrm{~min}$, rinsed 4-5 times with sterile water and incubated at room temperature in the dark for 3-5 days to synchronize germination. Spores were then plated on basal media $(1 / 2$ MS, pH 6.0) supplemented with $100 \mu \mathrm{g} \mathrm{ml}^{-1}$ ampicillin and maintained in humidity domes at $26^{\circ} \mathrm{C}$ with a light/ dark cycle of $16 / 8$ under light intensity of $100 \mu \mathrm{M} \mathrm{m}^{-2}$ $\mathrm{s}^{-1}$ for gametophyte development. Plates were inverted after 10 days of growth (d10) to deter fertilization. Sporophytes were grown in BLP germination soil \#1 (Beautiful Land Products, West Branch, IA) under humidity domes in the same light and temperature regime as gametophytes.

\section{Transformation of $C$. richardii gametophytes}

A 302-bp fragment (See Additional file 4: Table S1 for primer sequences) of $\mathrm{CrWOXB}$ was cloned into vectors pK7GWIWG2 and pH7GWIWG2 to generate $\mathrm{CrWOXB}$ RNAi constructs using the Gateway technology as described by Curtis and Grossniklaus [49] and Bui et al. [50]. Each construct was introduced into Agrobacterium tumefaciens strain GV3101 from Escherichia coli with an E.coli helper strain containing the pRK 2013 plasmid [51]. Stable transformation of young gametophyte tissue was conducted as described previously [52]. Successfully transformed gametophytes $\left(\mathrm{T}_{0}\right)$ were selected on media containing $50 \mu \mathrm{g} \mathrm{ml}^{-1}$ kanamycin or $5 \mu \mathrm{g} \mathrm{ml}^{-1}$ hygromycin. Resistant gametophytes were isolated and allowed to 
self-fertilize to produce sporophytes $\left(\mathrm{T}_{1}\right)$. Sporophytes were moved to liquid basal media and allowed to root before transplanting to soil. From the more than 20 independent transgenic lines isolated, 10 were chosen for qPCR analysis and characterization. Detailed phenotyping of three lines are presented here.

\section{Whole-mount and sectioned in situ hybridization}

Antisense and sense RNA probes used for in situ hybridization experiments were synthesized from $1 \mu \mathrm{g}$ of PCR products amplified using primers containing T7 promoter sequences (Additional file 4: Table S1) with T7 RNA polymerases (Agilent, Santa Clara, CA), and DIG RNA labeling mix (Roche Diagnostics, Indianapolis, IN). DIG-labeled RNA probes were precipitated in 2.25 $\mathrm{M} \mathrm{LiCl}$ overnight at $-20^{\circ} \mathrm{C}$, before resuspension in nuclease-free water. RNA concentration was measured with a Nanodrop One (Thermo-Scientific, Waltham, MA) and then diluted 1:1 with deionized formamide and stored at $-20^{\circ} \mathrm{C}$.

The SAM from young sporophytes with $10-11$ vegetative leaves, the youngest a visible fiddlehead, was dissected and vacuum infiltrated with fixing solution (4\% paraformaldehyde in 1x PBS) for 45 min and then incubated in fixing solution overnight at $4{ }^{\circ} \mathrm{C}$. Dehydration, embedding, pre-hybridization, hybridization and posthybridization washes were based on Jackson [59], except that acetic anhydride washes were omitted from prehybridization. Embedded tissues were sectioned at $8 \mu \mathrm{m}$ thickness with a rotary microtome. Probe detection and color development protocols were based on Ambrose et al. [58]. Whole-mount in situ was adapted from the protocol of Ambrose et al. [36, 58], with the following modifications. Gametophytes were fixed in FAA (formaldehyde: ethanol: acetic acid, 3.7\%:50\%:5\% v/v respectively) at room temperature for $1 \mathrm{~h}$, then stored in $70 \%$ ethanol at $-20^{\circ} \mathrm{C}$. Fixed gametophytes were processed without Histoclear II. Color development of wholemount in situ tissues was stopped in $\mathrm{ddH}_{2} \mathrm{O}$ and mounted in $50 \%$ glycerol. Whole-mount samples were viewed with a Zeiss compound light microscope and imaged with the Zeiss Axiocam ERc $5 \mathrm{~s}$ digital camera (Carl Zeiss Microscopy LLC, Thornwood, NY). DIC images of sectioned samples were viewed with a Nikon Eclipse E800 (Nikon Instruments Inc., Melville, NY) and captured with a Photometrics CoolSNAP cf. (Photometrics, Tucson, AZ). To confirm gene expression patterns, each in-situ experiment was repeated at least two times using different biological samples.

\section{RNA extraction and RT-PCR analyses}

Gametophyte and sporophyte tissue were harvested and flash frozen in liquid nitrogen, then stored at $-70^{\circ} \mathrm{C}$. Total RNA was extracted from frozen tissue with the
Quick-RNA MiniPrep (Plus) kit (Zymo Research, Irvine, CA) and $750 \mathrm{ng}$ of gametophyte total RNA or $500 \mathrm{ng}$ of sporophyte total RNA was used in reverse transcriptase reaction using MMLV (New England Biolabs, Ipswich, MA) with N9 random primers (IDT Coralville, IA). PCR was conducted with the following cycles: $2 \mathrm{~min}$ at $94{ }^{\circ} \mathrm{C}$, followed by 37 cycles of $30 \mathrm{~s}$ at $94{ }^{\circ} \mathrm{C}, 30$ s at $59^{\circ} \mathrm{C}$, and 30 s at $72{ }^{\circ} \mathrm{C}$, with a $5 \mathrm{~min}$ final extension time at $72{ }^{\circ} \mathrm{C}$ for $C r W O X B$, and 25 cycles under the same conditions for $C r U B Q$ transcripts.

For RT-qPCR, three biological and two technical replicates were performed for each line. Total RNA from whole young sporophytes with 6-7 fully expanded round leaves was extracted and $200 \mathrm{ng}$ were used in cDNA synthesis as described above. Due to delay in development of crwoxb lines, the age of the sporophytes in both the wild-type and crwoxb lines was determined by numbers of leaves and not days. Primers for qPCR are listed in Additional file 4: Table S1. Detection of amplification was performed using SYBR green chemistry (Roche Diagnostic, Indianapolis, IN) with the Roche LightCycler 480 Real-Time PCR system (Roche Diagnostic). The PCR cycle was as follows: $10 \mathrm{~min}$ at $95^{\circ} \mathrm{C}$, followed by $45-55$ cycles of $10 \mathrm{~s}$ at $95^{\circ} \mathrm{C}, 10 \mathrm{~s}$ at $62^{\circ} \mathrm{C}$, and $20 \mathrm{~s}$ at $72{ }^{\circ} \mathrm{C}$, with a single fluorescence read at the end of each extension time. A melting curve analysis was also performed and analyzed using the $\mathrm{Tm}$ calling software module to verify the absence of primer dimers and nonspecific products. Calibrator normalized relative quantification was performed using the 2nd derivative maximum algorithm with three internal relative standards. $C r W O X B$ expression was measured relative to $C r U B Q$.

\section{Phenotypic analysis of crwoxb lines}

To count cells of the gametophytes, gametophytes were cleared overnight in $100 \%$ ethanol at $4{ }^{\circ} \mathrm{C}$, then rinsed 3 times for $5 \mathrm{~min}$ in water and stained with Hoechst 33342 $\left(40 \mu \mathrm{g} \mathrm{ml}^{-1}\right)$ (Invitrogen, Carlsbad, CA) for at least 15 min, rinsed in water and mounted on slides with $50 \%$ glycerol. Gametophytes were then imaged with a Leica stereomicroscope and a Qicam camera (Qimaging, Surrey, BC, Canada) with a DAPI filter. Nuclei of gametophytes were counted in Photoshop CC (Adobe systems, San Jose, CA). Brightness and contrast were increased slightly to facilitate cell counting.

For root and lateral root counts, spores of wild-type and crwoxb lines were grown on basal media for 13 days, after which individual hermaphroditic gametophytes were isolated for self-fertilization by adding a few drops of water. Resulting sporophytes were transferred to 100 $\mathrm{ml}$ of liquid basal media with $100 \mu \mathrm{g} \mathrm{ml}^{-1}$ ampicillin and grown for an additional 2 weeks before roots and lateral roots were counted. Vegetative leaves and pinnae were 
counted on soil-grown sporophytes when each sporophyte had 5-7 sporophylls.

\section{Statistical evaluation of the data}

Statistical analyses of CrWOXB levels in crwoxb lines, gametophyte archegonia numbers, and sporophyte phenotypes were conducted with one-way ANOVA, while gametophyte cell numbers were conducted with twoway ANOVA. Both analyses were followed by Dunnett's multiple comparisons test. All calculations were done in GraphPad Prism version 8.0.1 (GraphPad Software, San Diego, CA).

\section{Phylogeny of WOX proteins}

Multiple sequence alignments of WOX homeodomains are based on T-Coffee [53] and trees were built using the Maximum-Likelihood method in phyML [54] with 500 bootstrap replicates and visualized in MEGA7 [55]. Protein sequences for Ostreococcus tauri, Osctreococcus lucimarinus, Physcomitrella patens, Selaginella kraussiana, Oryza sativa were obtained from Phytozome [56]. Azolla filiculoides, Salvinia cuculata sequences were obtained from Fernbase [57]. Ceratopteris richardii sequences were obtained from NCBI. Arabidopsis thaliana sequence were obtained from TAIR. Full length protein sequences are provided in Additional file 7.

\section{Additional files}

Additional file 1: Figure S1. Phylogeny of WOX proteins from representative embryophytes. Abbreviations Ot, Ostreococcus tauri; Ol, Osctreococcus lucimarinus; Pp, Physcomitrella patens; Sk, Selaginella kraussiana; Af, Azolla filiculoides; Sc, Salvinia cuculata; Cr, Ceratopteris richardii; Os, Oryza sativa; At, Arabidopsis thaliana. Evolutionary history was inferred using Maximum-likelihood method with 500 bootstrap replicates as a test of relatedness. Alignment of sequences was conducted with TCoffee and cladogram constructed in phyML and visualized with MEGA7. The WOX genes in the two sequenced fern genomes, Azolla and Salvinia [58] have not been previously included in constructing any WOX tree. Asterisk denote outgroup. (DOCX $1484 \mathrm{~kb}$ )

Additional file 2: Figure S2. Bright-field microscopy of d13 transgenic gametophytes. (a) RN3 (wild type). (b-d) transgenic gametophytes. (a-d inset) $2 \times$ digital-enlargement of a $\sim 250 \times 250$ pixel region of the notch meristem. Red lines outline the notch meristem, in (a) the prothallus overlaps and no space is present between lobes of the prothallus. Scale bar $=0.5 \mathrm{~mm}$. (DOCX $12696 \mathrm{~kb})$

Additional file 3: Figure S3. Protein domain comparison of intermediate clade WOX proteins from A. thaliana and C. richardii. (a) Model of intermediate clade WOX proteins from $C$. richardii and $A$. thaliana, green boxes represent the homeodomain and blue the $C$ terminal domain. (b) T-Coffee sequence alignment of $C$. richardii and $A$. thaliana intermediate WOX proteins domains. Specific motifs are outlined, and conserved residues are highlighted in green, less conserved sites are highlighted in yellow. (DOCX $160 \mathrm{~kb}$ )

Additional file 4: Table S1. Primer sequences used in the study. Sequences left of * are added for directional cloning or T7 promoter sequence. All sequences are 5' to 3'. (DOCX $20 \mathrm{~kb}$ )

Additional file 5: Table S2. Average number of gametophyte cells observed at days 7 to 10 of growth and the average number of cells produced per day. ${ }^{*}, p<0.05 ;{ }^{* *}, p<0.01 ;{ }^{* * *}, p<0.001 ;{ }^{* * * *}, p<0.0001$, Two-way ANOVA. (DOCX $20 \mathrm{~kb}$ )

Additional file 6: Table S3. Transgenic and wild-type gametophyte cell and archegonia numbers at d13. ${ }^{* * *}, p<0.0001$. Two-way ANOVA, cell numbers or One-way ANOVA, archegonium numbers. (DOCX 20 kb)

Additional file 7: Sequences used in Phylogeny. (TXT $20 \mathrm{~kb}$ )

\section{Abbreviations}

CZ: Central zone; DAPI: 4',6-diamidino-2-phenylindole; DIG: Digoxygenin; FAA: Formaldehyde: ethanol: acetic acid; mRNA: Messenger RNA; MS: Murashige and Skoog; NCBI: National Center for Biotechnology Information; OC: Organizing center; PBS: Phosphate buffered saline; RAM: Root apical meristem; RNAi: RNA interference; RT-PCR: Reverse transcription polymerase chain reaction; RT-qPCR or qPCR: Reverse transcription - quantitative polymerase chain reaction; SAM: Shoot apical meristem; TAIR: The Arabidopsis Information Resource; UBQ: Ubiquitin; WOX: WUSCHEL-related homeobox; WUS: WUSCHEL

\section{Acknowledgements}

We have the permission to thank Linh Bui (Indiana University, Bloomington, IN) for initiating the project, Angela R. Cordle and Kelley Withers for critical reading of the manuscript.

\section{Authors' contributions}

$\mathrm{CY}$ designed and carried out the experiments, analyzed data and drafted the manuscript. LG helped count gametophyte cell numbers. El provided input for the project, revised and critically edited the manuscript. CLC supervised the research, drafted and edited the manuscript. All authors read and approved the final version of the manuscript.

\section{Funding}

The work was supported by UI Research Council and NSF1555487. CY was also supported by the Avis Cone Summer Fellowship and UI Graduate Summer Fellowship. The funding bodies had no role in the design of the study or analysis, interpretation of data, or writing the manuscript.

Availability of data and materials

See "Phylogeny of WOX proteins" section for the datasets used and/or analyzed during the current study.

Ethics approval and consent to participate

Not applicable.

\section{Consent for publication}

Not applicable.

\section{Competing interests}

The authors declare that they have no competing interests.

Received: 28 May 2019 Accepted: 27 August 2019

Published online: 11 October 2019

\section{References}

1. Groß-Hardt R, Laux T. Stem cell regulation in the shoot meristem. J Cell Sci. 2003;116(9):1659-66.

2. Tucker MR, Laux T. Connecting the paths in plant stem cell regulation. Trends Cell Bio. 2007;17(8):403-10.

3. Barton MK. Twenty years on: the inner workings of the shoot apical meristem, a developmental dynamo. Dev Biology. 2010;341(1):95-113.

4. Galli M, Gallavotti A. Expanding the regulatory network for meristem size in plants. Trends Genet. 2016;32(6):372-83.

5. Somssich M, Je BI, Simon R, Jackson D. CLAVATA-WUSCHEL signaling in the shoot meristem. Development. 2016;143(18):3238.

6. Jung JKH, McCouch S. Getting to the roots of it: genetic and hormonal control of root architecture. Front Plant Sci. 2013;4:186

7. Hepworth SR, Pautot VA. Beyond the divide: boundaries for patterning and stem cell regulation in plants. Front Plant Sci. 2015;6:1052.

8. Yadav RK, Perales M, Gruel J, Girke T, Jonsson H, Reddy GV. WUSCHEL protein movement mediates stem cell homeostasis in the Arabidopsis shoot apex. Genes Dev. 2011;25(19):2025-30. 
9. Haecker A, Gross-Hardt R, Geiges B, Sarkar A, Breuninger H, Herrmann M, et al. Expression dynamics of WOX genes mark cell fate decisions during early embryonic patterning in Arabidopsis thaliana. Development. 2004; 131(3):657-68.

10. van der Graaff E, Laux T, Rensing SA. The WUS homeobox-containing (WOX) protein family. Genome Biol. 2009;10(12):248.

11. Nardmann J, Werr W. The invention of WUS-like stem cell-promoting functions in plants predates leptosporangiate ferns. Plant Mol Bio. 2012; 78(1):123-34.

12. Deveaux $Y$, Toffano-Nioche $C$, Claisse $G$, Thareau V, Morin H, Laufs P, et al. Genes of the most conserved WOX clade in plants affect root and flower development in Arabidopsis. BMC Evol Biol. 2008;8:291.

13. Lian G, Ding Z, Wang Q, Zhang D, Xu J. Origins and evolution of WUSCHELrelated Homeobox protein family in plant kingdom. Sci World J. 2014;2014: 534140

14. Ge $Y$, Liu J, Zeng $M, H e J$, Qin $P$, Huang $H$, et al. Identification of WOX family genes in Selaginella kraussiana for studies on stem cells and regeneration in lycophytes. Front Plant Sci. 2016;7:93.

15. Hedman H, Zhu T, von Arnold S, Sohlberg JJ. Analysis of the WUSCHELRELATED HOMEOBOX gene family in the conifer Picea abies reveals extensive conservation as well as dynamic patterns. BMC Plant Biol. 2013;13:89.

16. Dolzblasz A, Nardmann J, Clerici E, Causier B, van der Graaff E, Chen J, et al. Stem cell regulation by Arabidopsis WOX genes. Mol Plant. 2016;9(7):1028-39.

17. Laux T, Mayer KF, Berger J, Jurgens G. The WUSCHEL gene is required for shoot and floral meristem integrity in Arabidopsis. Development. 1996; 122(1):87-96.

18. Sarkar AK, Luijten M, Miyashima S, Lenhard M, Hashimoto T, Nakajima K, et al. Conserved factors regulate signalling in Arabidopsis thaliana shoot and root stem cell organizers. Nature. 2007:446(7137):811-4.

19. Kamiya N, Nagasaki H, Morikami A, Sato Y, Matsuoka M. Isolation and characterization of a rice WUSCHEL-type homeobox gene that is specifically expressed in the central cells of a quiescent center in the root apical meristem. Plant J. 2003:35(4):429-41.

20. Suer S, Agusti J, Sanchez P, Schwarz M, Greb T. WOX4 imparts auxin responsiveness to cambium cells in Arabidopsis. Plant Cell. 2011:23(9):3247-59.

21. Hirakawa $Y$, Kondo $Y$, Fukuda $H$. TDIF peptide signaling regulates vascular stem cell proliferation via the WOX4 homeobox gene in Arabidopsis. Plant Cell. 2010;22(8):2618-29.

22. Shimizu R, Ji J, Kelsey E, Ohtsu K, Schnable PS, Scanlon MJ. Tissue specificity and evolution of meristematic WOX3 function. Plant Physiol. 2009;149(2): 841-50.

23. Nakata M, Matsumoto N, Tsugeki R, Rikirsch E, Laux T, Okada K. Roles of the middle domain-specific WUSCHEL-RELATED HOMEOBOX genes in early development of leaves in Arabidopsis. Plant Cell. 2012;24(2):519-35.

24. Ji J, Shimizu R, Sinha N, Scanlon MJ. Analyses of WOX4 transgenics provide further evidence for the evolution of the WOX gene family during the regulation of diverse stem cell functions. Plant Sign B. 2014;5(7):916-20.

25. Breuninger $\mathrm{H}$, Rikirsch $\mathrm{E}$, Hermann M, Ueda M, Laux T. Differential expression of WOX genes mediates apical-basal axis formation in the Arabidopsis embryo. Dev Cell. 2008;14(6):867-76.

26. Ueda M, Zhang Z, Laux T. Transcriptional activation of Arabidopsis axis patterning genes WOX8/9 links zygote polarity to embryo development. Dev Cell. 2011;20(2):264-70.

27. Wu X, Dabi T, Weigel D. Requirement of homeobox gene STIMPY/WOX9 for Arabidopsis meristem growth and maintenance. Curr Biol. 2005;15(5):436-40.

28. Romera-Branchat M, Ripoll JJ, Yanofsky MF, Pelaz S. The WOX13 homeobox gene promotes replum formation in the Arabidopsis thaliana fruit. Plant J. 2013;73(1):37-49

29. Yasui Y, Ohmori Y, Takebayashi Y, Sakakibara H, Hirano H-Y. WUSCHELRELATED HOMEOBOX4 acts as a key regulator in early leaf development in rice. PLoS Genet. 2018;14(4):e1007365.

30. Zhu T, Moschou PN, Alvarez JM, Sohlberg JJ, von Arnold S. Wuschel-related homeobox 8/9 is important for proper embryo patterning in the gymnosperm Norway spruce. J Exp Bot. 2014;65(22):6543-52.

31. Zhu T, Moschou PN, Alvarez JM, Sohlberg JJ, von Arnold S. WUSCHELRELATED HOMEOBOX 2 is important for protoderm and suspensor development in the gymnosperm Norway spruce. BMC Plant Biol. 2016; 16(1):19.

32. Sakakibara $K$, Reisewitz $P$, Aoyama T, Friedrich $T$, Ando $S$, Sato $Y$, et al. WOX13-like genes are required for reprogramming of leaf and protoplast cells into stem cells in the moss Physcomitrella patens. Development. 2014; 141(8):1660-70.

33. Nardmann J, Werr W. Symplesiomorphies in the WUSCHEL clade suggest that the last common ancestor of seed plants contained at least four independent stem cell niches. New Phytol. 2013;199(4):1081-92.

34. Zhou X, Guo Y, Zhao P, Sun M-X. Comparative Analysis of WUSCHEL-Related Homeobox Genes Revealed Their Parent-of-Origin and Cell Type-Specific Expression Pattern During Early Embryogenesis in Tobacco. Front Plant Sci. 2018;9:311.

35. Stevenson D. The Cytohistological and Cytohistochemical zonation of the shoot apex of Botrychium multifidum. Am J Bot. 1976;63(6):852-6.

36. Ambrose BA, Vasco A. Bringing the multicellular fern meristem into focus. New Phytol. 2016;210(3):790-3.

37. Gelvin SB. Agrobacterium-mediated plant transformation: the biology behind the "gene-jockeying" tool. Microbiol Mol Biol Rev. 2003;67(1):16-37.

38. Döpp W. Über eine hemmende und eine fördernde Substanz bei der Antheridienbildung in den Prothallien von Pteridium aquilinum. Berichte de Deutschen Botanischen Gesellschaft. 1959;72(1):11-24.

39. Peng L, Skylar A, Chang PL, Bisova K, Wu X. CYCP2;1 integrates genetic and nutritional information to promote meristem cell division in Arabidopsis. Dev Biol. 2014:393(1):160-70.

40. Wu X, Chory J, Weigel D. Combinations of WOX activities regulate tissue proliferation during Arabidopsis embryonic development. Dev Biol. 2007; 309(2):306-16.

41. Banks JA. Gametophyte development in ferns. Annu Rev Plant Biol. 1999;50:163-86.

42. Bartz M, Gola EM. Meristem development and activity in gametophytes of the model fern, Ceratopteris richardii. Dev Biol. 2018;444(2):107-15.

43. Skylar A, Hong F, Chory J, Weigel D, Wu X. STIMPY mediates cytokinin signaling during shoot meristem establishment in Arabidopsis seedlings. Development. 2010;137(4):541-9.

44. Szövényi P, Rensing SA, Lang D, Wray GA, Shaw AJ. Generation-biased gene expression in a bryophyte model system. Mol Biol Evol. 2011;28(1):803-12.

45. Hou G, Hill JP. Heteroblastic root development in Ceratopteris richardi (Parkeriaceae). Int J Plant Sci. 2002;163(3):341-51.

46. Hill JP. Meristem development at the Sporophyll Pinna apex in Ceratopteris richardii. Int J Plant Sci. 2001;162(2):235-47.

47. Sanders HL, Darrah PR, Langdale JA. Sector analysis and predictive modelling reveal iterative shoot-like development in fern fronds. Development. 2011;138(14):2925-34.

48. Hou G, Hill JP, Blancaflor EB. Developmental anatomy and auxin response of lateral root formation in Ceratopteris richardii. J Exp Bot. 2004;55(397):685-93.

49. Curtis MD, Grossniklaus U. A gateway cloning vector set for high-throughput functional analysis of genes in planta. Plant Physiol. 2003;133(2):462-9.

50. Bui LT, Pandzic D, Youngstrom CE, Wallace S, Irish EE, Szövényi $P$, et al. A fern AINTEGUMENTA gene mirrors BABY BOOM in promoting apogamy in Ceratopteris richardii. Plant J. 2017;90(1):122-32

51. Hausner M, Wuertz S. High rates of conjugation in bacterial biofilms as determined by quantitative In Situ analysis. Appl and Environ Microb. 1999; 65(8):3710-3.

52. Bui LT, Cordle AR, Irish EE, Cheng C-L. Transient and stable transformation of Ceratopteris richardii gametophytes. BMC Res Notes. 2015:8:214-24.

53. Notredame C, Higgins DG, Heringa J. T-coffee: a novel method for fast and accurate multiple sequence alignment11Edited by J. Thornton J Mol Bio. 2000;302(1):205-17.

54. Guindon S, Dufayard J-F, Lefort V, Anisimova M, Hordijk W, Gascuel O, New algorithms and methods to estimate maximum-likelihood phylogenies: assessing the performance of PhyML 3.0. Syst Biol. 2010:59(3):307-21.

55. Stecher G, Kumar S, Tamura K. MEGA7: molecular evolutionary genetics analysis version 7.0 for bigger datasets. Mol Bio Evol. 2016;33(7):1870-4

56. Phytozome. Department of Energy's Joint Genome Institute. 2017 https:// phytozome.jgi.doe.gov/pz/portal.html. Accessed 5 Mar 2019.

57. Li F-W, Brouwer P, Carretero-Paulet L, Cheng S, de Vries J, Delaux P-M, et al. Fern genomes elucidate land plant evolution and cyanobacterial symbioses. Nat Plants. 2018:4(7):460-72.

58. Ambrose BA, Lerner DR, Ciceri P, Padilla CM, Yanofsky MF, Schmidt RJ. Molecular and genetic analyses of the Silkyl gene reveal conservation in floral organ specification between eudicots and monocots. Mol Cell. 2000; 5(3):569-79.

59. Jackson D. In situ hybridization in plants. In: Bowles DJ, McPherson MJ, Gurr SJ, editors. Molecular plant pathology: a practical approach. 1. New York: IRL Press at Oxford University Press; 1992. p. 163-74. 
60. Hagemann W. Morphological aspects of leaf development in ferns and angiosperms. In: White RA, Dickinson WC, editors. Contemporary Problems in Plant Anatomy. Orlando, FL: Academic Press; 1984. p. 301-49.

\section{Publisher's Note}

Springer Nature remains neutral with regard to jurisdictional claims in published maps and institutional affiliations.

Ready to submit your research? Choose BMC and benefit from:

- fast, convenient online submission

- thorough peer review by experienced researchers in your field

- rapid publication on acceptance

- support for research data, including large and complex data types

- gold Open Access which fosters wider collaboration and increased citations

- maximum visibility for your research: over $100 \mathrm{M}$ website views per year

At BMC, research is always in progress. 